\title{
Endodontic Management of Separated Instrument in Mandibular First Molar- A Case Report
}

\author{
Dr. Ankush Jasrotia, ${ }^{1}$ Dr. Nidhi Sharma, ${ }^{2}$ \\ ${ }^{1}$ M.D.S. in Conservative Dentistry \& Endodontics, Malvika Dental Clinic, Kathua, 9018091399.) \\ ${ }^{21}$ B.D.S. Malvika Dental Clinic, Kathua, 9906232299.)
}

\begin{abstract}
With the increased practice of rotary endodontics in recent years, separated rotary nickel - titanium (NiTi) files in root canals is the most commonly reported mishap, causing lot of stress and anxiety among clinicians and patients. In some cases, removal of a fractured instrument is impossible or undesirable. Although integration of modern techniques into endodontic practice has improved the clinician's ability to retrieve separated instrument but removal may not always be possible due to many factors like poor access, visibility, location of the instrument and the amount of dentin to be removed. While keeping these factors in mind, bypassing technique is more effective and conservative. The aim of this paper is to present a clinical case in which a separated instrument was bypassed inside the curvature of distobuccal root canal of mandibular first molar during endodontic treatment.
\end{abstract}

Keywords: Separated instrument, bypass, small files, warm vertical compaction.

\section{Introduction}

The presence of a separated instrument in the root canal may lead to a failure of the treatment of the tooth. The prognosis depends on the degree of contamination of the canal at the moment of separation and the presence of apical pathology. The probability of removing a fractured instrument is directly connected to visibility - i.e. possibility to create straight line access to it. When the fragment is located inside or beyond the root canal's curvature, visibility requires straightening of the root canal to a different extent, which may lead to removing excessive amount of dentin and root weakening or even perforation. That's why such cases require very good assessment of the risk and indications for the procedure.[1, 2, 3, 4]

An alternative technique, which historically precedes those for broken instruments removal, is the so called, "bypass". Inserting a fine file between the fragment and the root canal wall may lead to negotiating the canal to full working length and enable thorough instrumentation and root canal obturation with the fragment remaining in situ. Incorporating the fragment in the root canal obturation material considerably improves the case prognosis.[5] This technique does not require direct visibility to the fragment- i.e. it can be suitable for when the fragment is located beyond a considerable root canal curvature.

\section{Case Report}

A 14-years old male patient was referred to my clinic without any history of systemic disease complaining of severe pain in the left lower molar tooth since one week. The referring dentist had already performed preliminary root canal treatment. Vitality tests on tooth showed painful response to hot, cold and electrical pulp tests. Before starting the treatment, a new diagnostic radiograph was taken (Fig1). It showed fractured instrument in the curvature of distal root of left mandibular first molar. From clinical and radiographic findings, a diagnosis of symptomatic irreversible pulpitis was made. Treatment options were discussed with the patient and endodontic therapy was the treatment of choice. The tooth was anesthetised with $1.8 \mathrm{ml} 2 \%$ lignocaine containing 1:200,000 adrenaline followed by rubber dam isolation. Access cavity was modified to locate all canal orifices and finally four canal orifices were found- mesiobuccal, mesiolingual, distobuccal and distolingual. While cleaning and shaping the canals, a resistance was felt by 8 no.K file in the curvature of distobuccal canal.

Since the fractured segment could not be visualised and was below the root curvature, bypassing was preferred over retrieval. The access cavity was filled with chelating agent - 17\% EDTA (Avuprep) and a no.6 K file was introduced into the canal for searching a way to bypass the instrument. After a few tries, it was able to get the $6 \mathrm{~K}$ file past the instrument. Working length was established with the help of an apex locator (Root ZX, $\mathrm{J}$ Morita Inc: USA). During the shaping of canals, copious irrigation with $5.25 \%$ sodium hypochlorite and saline was performed. Patency was kept with an 8 size K file between every instrument. Shaping of all four canals were done upto \#0.04/25 Revo S rotary files (Micro Mega, France). Canals were irrigated with $5.25 \%$ sodium hypochlorite, $17 \%$ EDTA and $2 \%$ chlorhexidine. Canals were dried using paper points and a master cone radiograph was taken (Fig 2) to check the apical fit in all the four canals. Finally canals were obturated with 
gutta-percha and AH plus sealer (Dentsply, Detrey, Konstaz, Germany) by warm vertical compaction (Fig 3). The access preparation was sealed and the post endodontic restoration was planned.

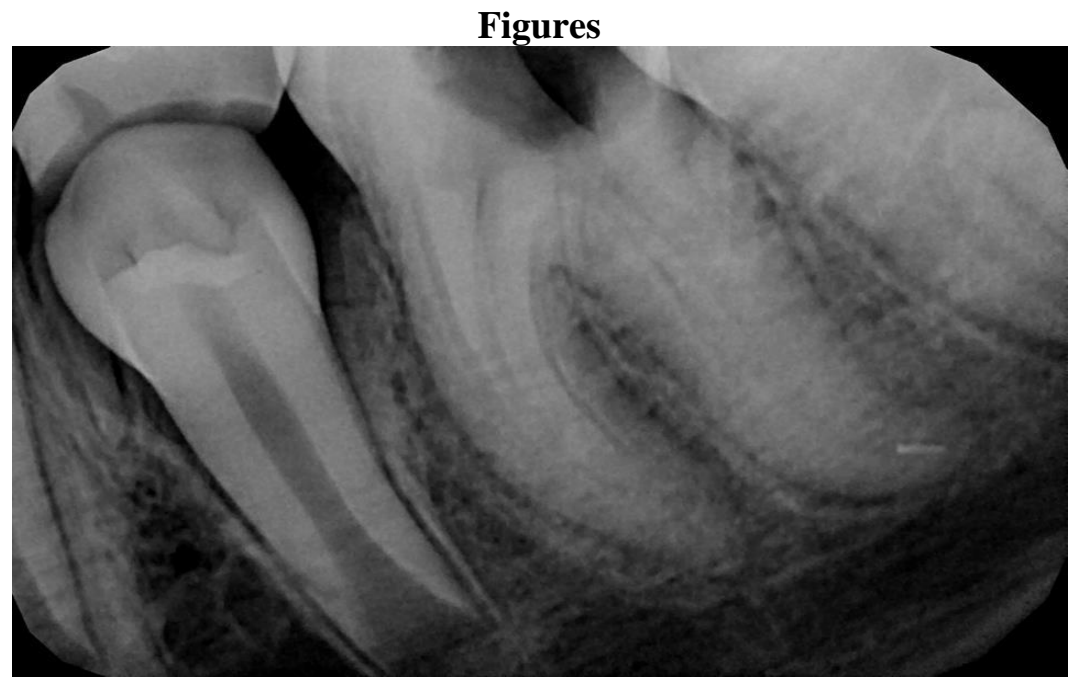

Fig 1. preoperative radiograph of mandibular first molar showing fractured file in distal root.

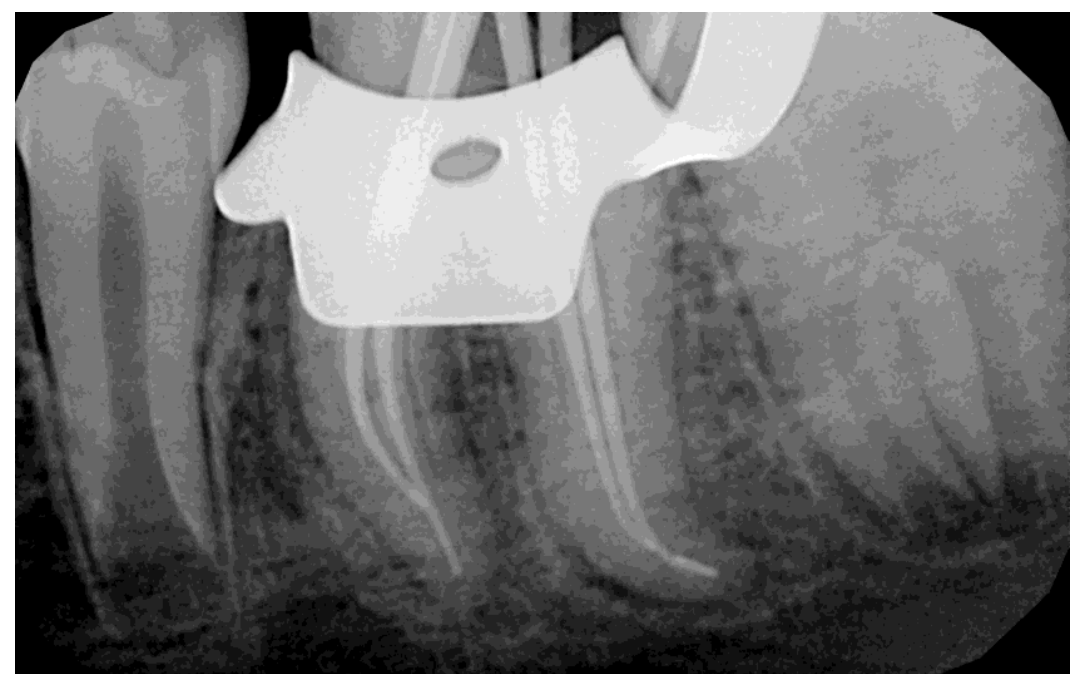

Fig 2: master cone radiograph after bypassing the fractured instrument in the distobuccal root canal

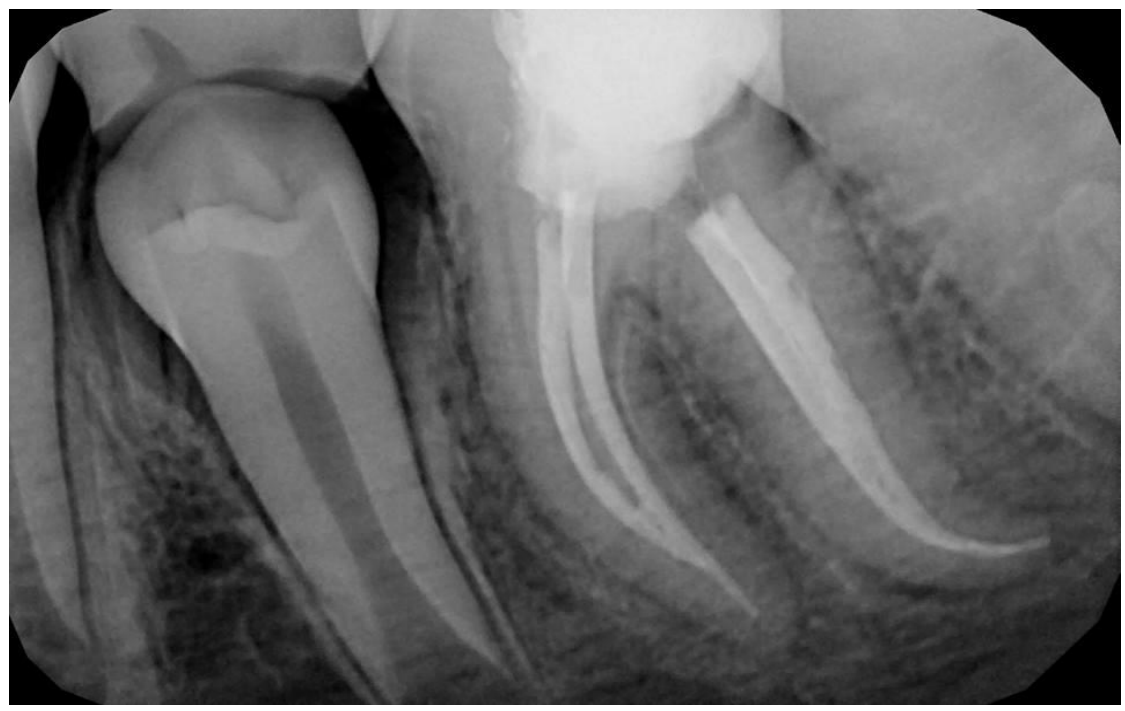

Fig 3: post obturation radiograph. 


\section{Discussion}

Separated root canal instruments are one of the most troublesome incidents in endodontic therapy, especially if the tooth is non-vital and fragment cannot be removed. In the majority of cases, the procedural mishap does not directly compromise the prognosis, unless a concomitant infection is already present. Separated fragment of instrument in root canal may be indirectly responsible for an endodontic failure by limiting the access to the apical part of the canal, compromising disinfection and obturation, but is rarely directly responsible.[6]

Two studies reported that the retained fragment reduced healing, particularly in the presence of a preexisting periapical radiolucency[7, 8] whereas another two studies stated that it had no influence on healing.[9] According to some authors, the retained fractured instrument can be incorporated into the final root canal filling.[10]

The four treatment protocols have been suggested by the literature for management of fractured instruments in root canals:

1. Allowing the separated instrument to be retained in the canal and treating the remaining portion of canal.

2. Bypassing the separated fragment and treating the canal.

3. Retrieving the separated fragment and treating the canal.

4. Surgical approach for retrieval of separated fragment followed by treatment accordingly.

Favourable factors for the removal of a fractured instrument are straight canals, incisors and canines; localisation before the curvature; length of fragment of more than $5 \mathrm{~mm}$; localisation in the coronal or middle third of the root canal; reamer or lentulo spirals; and hand NiTi K-files. As in present case report the separated instrument is located inside the root canal curvature, visibility requires straightening of the root canal to a different extent, which may lead to removing excessive amount of dentin and root weakening or even perforation. $[1,2,3,4]$ The decision was made to try to bypass the instrument rather than try to retrieve it. Thermoplasticized obturation techniques were suggested in these cases since they seal the gap between the fractured fragment and canal walls better than other techniques like cold lateral compaction, single greater tapered cone obturation, because of their excellent flow.[11]

\section{Conclusion}

Management of instrument separation is a complex issue. Under ideal conditions it is preferable to remove the fragment and pursue treatment, but this is not always possible. The risks of removal should be balanced against benefits, as weakening of the tooth or perforation during instrument removal may be more detrimental than the fragment of instrument.

\section{References}

[1]. Ruddle CJ. Non-surgical retreatment. J Endod, 30(12), 2004 Dec, 827-45.

[2]. Ruddle CJ. Broken instrument removal. The endodontic challenge. Dent Today,21(7),2002 Jul, 70-72, 74, 76.

[3]. Ruddle CJ. Non-surgical retreatment. In: Cohen S, Burns RC, eds. Pathways of the pulp, 8th ed. St Louis: Mosby, 2002, 875-930.

[4]. Ruddle CJ. Micro-endodontic non-surgical retreatment. DentClinNorth Am, 41(3), 1997 Jul, $429-54$.

[5]. Saunders JL, Eleazer PD, ZhangP, Michalek S. Effect of a separated instrument on bacterial penetration of obturated root canals. J Endod 30(3),2004 Mar, 177-79.

[6]. Siqueira JF, Jr. Aetiology of root canal treatment failure: why well-treated teeth can fail. IntEndod J, 34, 2001, 1-10.

[7]. Strindberg LZ. The dependence of the results of pulp therapy on certain factors: an analytical study based on radiographic and clinical follow-up examinations. ActaOdontolScand, 14(Suppl 21), 1956, 1-175.

[8]. Grossman LI. Guidelines for the prevention of fracture of root canal instruments. Oral Surg Oral Med Oral Pathol, 28,1969, 746 52.

[9]. Ingle JI, Glick D. The Washington study. In: Ingle JI, ed. Endodontics, 1st ed. Philadelphia: Lea \& Febiger, 1965,54 -77.

[10]. Crump MC, Natkin E. Relationship of broken root canal instruments to endodontic case prognosis: a clinical investigation. J Am Dent Assoc, 80,1970, 1341-7.

[11]. Taneja S, Chanda R, Gupta R,Gupta A. Comparitive evaluation of sealing properties of different obturation systems placed over apically fractured rotary NiTi files. J Conserv. Dent, 15, 2005, 36-40. 